Received: 10.06 .2019

Revised: 28.06 .2019

Accepted: 28.06 .2019

DOI: $10.17804 / 2410-9908.2019 .3 .041-051$

\title{
EVOLUTION OF THE STRUCTURE OF TIN BRONZE AND COPPER AFTER DYNAMIC COMPRESSION BY THE KOLSKY METHOD USING A SPLIT HOPKINSON PRESSURE BAR
}

\author{
A. V. Stolbovsky ${ }^{1, a)^{*}}$, V. V. Popov ${ }^{1,2, b)}$, R. M. Falakhutdinov ${ }^{1, c)}$, S. A. Murzinova, ${ }^{1, d}$, \\ E. V. Shorokhov ${ }^{3}$, Z. I. Zavyalov ${ }^{3}$, A. A. Degtyarev ${ }^{3}$ \\ ${ }^{I}$ M.N. Miheev Institute of Metal Physics, UB RAS, 18 S. Kovalevskoy St., Ekaterinburg, 620108, Russian Federation \\ ${ }^{2}$ Ural State University of Economics, 62 8-Marta St., Ekaterinburg, 620144, Russian Federation a \\ ${ }^{3}$ Russian Federal Nuclear Center - VNIITF, Snezhinsk, Russian Federation \\ a) (iD http://orcid.org/0000-0001-5228-7857 @ stolbovsky@imp.uran.ru; \\ b) (iD http://orcid.org/0000-0002-9291-0102 《 vpopov@imp.uran.ru; \\ c) (iD http://orcid.org/0000-0001-8505-9455 \&alahutdinov@imp.uran.ru; \\ d) (iD http://orcid.org/0000-0001-5240-2966 @ murzinova@imp.uran.ru \\ *Corresponding author. E-mail: stolbovsky@imp.uran.ru \\ Address for correspondence: 18 S. Kovalevskoy St., Ekaterinburg, 620108, Russian Federation \\ Tel.: +7 (343) 37835 74; fax: (343) 3745244
}

The effect of high-speed dynamic compression on the structure and mechanical properties of low-alloyed tin bronze in different initial states (coarse-grained, with additional annealing, and cast, obtained by spun casting) and commercially pure copper of the M1 standard is investigated. The results obtained show that the dynamic compression of tin bronze samples in the coarse-grained initial state causes intensive twinning starting from a strain rate of $2.6 \cdot 10^{3} \cdot \mathrm{s}^{-1}$. At relatively low deformation rates, the dynamic compression of samples in the initial cast state promotes an increase in their strength characteristics, whereas, with a further strain rate increase, the development of relaxation processes is observed. However, additional homogenization annealing decreases the probability of relaxation processes under loading, which results in the highest possible mechanical characteristics. The studies of the M1 commercially pure copper have shown that variations in the deformation rate much less affect the properties than in case of bronze, and their values are lower due to relaxation processes both under loading and after deformation.

Keywords: plastic deformation, high-speed deformation, grain boundaries, thermal stability, dynamic compression, Kolsky method, split Hopkinson pressure bar, tin bronze, copper.

\section{Acknowledgments}

The work was performed within the framework of the state assignment from the Russian Ministry of Education and Science (theme Function, state registration number AAAA-A19-119012990095-0) and supported by the UB RAS Basic Research Program (project No. 18-10-2-37).

\section{References}

1. Stolbovsky A.V., Popov V.V., Popova E.N. Structure and Thermal Stability of Tin Bronze Nanostructured by High Pressure Torsion. Diagnostics, Resource and Mechanics of materials and structures, 2015, iss. 5, pp. 118-132. DOI: 10.17804/2410-9908.2015.5.118-132. Available at: http://dream-journal.org/issues/2015-5/2015-5_52.html (accessed: 30.10.2017). 
2. Stolbovsky A.V., Popov V.V., Popova E.N., Falahutdinov R.M. Effect of Severe Plastic Deformation by High-Pressure Torsion on the Structure and Properties of Copper and Tin Bronze. Diagnostics, Resource and Mechanics of materials and structures, 2017, iss. 5, pp. 13-22. DOI: 10.17804/2410-9908.2017.5.013-022. Available at: http://dream-journal.org/issues/20175/2017-5_144.html (accessed: 09.07.2018).

3. Khomskaya I.V., Zel'dovich V.I., Shorokhov E.V., Frolova N.Yu., Zhgilev I. N., Kheifets A.E. Structure Formation in Copper during Dynamic Channel_Angular Pressing. Phys. Met. Metallogr., 2008, vol. 105, no. 6, pp. 586-593. DOI: 10.1134/S0031918X08060094.

4. Shorokhov E.V., Zhgilev I.N., Khomskaya I.V., Brodova I.G., Zel'dovich V.I., Gunderov D.V., Frolova N.Yu., Gurov A.A., Oglezneva N.P., Shirinkina I.G., Kheifets A.E., Astaf'ev V.V. High Speed Deformation of Metallic Materials Using Channel Angular Pressing for Producing an Ultrafine Grained Structure. Russ. Metall. (Metally), 2010, no. 4, pp. 323-327. DOI: $10.1134 / \mathrm{S} 0036029510040142$.

5. Khomskaya I.V., Zel'dovich V.I., Kheifets A.E., Frolova N.Yu., Dyakina V.P., Kazantsev V.A. Evolution of the Structure upon Heating of Submicrocrystalline and Nanocrystalline Copper Produced by High Rate Deformation. Phys. Met. Metallogr., 2011, vol. 111, no. 4, pp. 367-374. DOI: $10.1134 /$ S0031918X11030070.

6. $\quad$ Stolbovsky A.V., Popov V.V., Popova E.N., Falahutdinov R.M., Shorohov E.V. Specific features of strain behavior of bronzes under dynamic channel-angular pressing. International Journal of Applied and Fundamental Research, 2016, no. 11-5, pp. 858-863. (In Russian).

7. Petrova A.N., Brodova I.G., Razorenov S.V. Strength properties and structure of a submicrocrystalline Al-Mg-Mn alloy under shock compression. Phys. Met. Metallogr., 2017, vol. 118, no. 6, pp. 601-607. DOI: 10.1134/S0031918X17060072.

8. Garkushin G.V., Ivanchikhina G.E., Razorenov S.V., Ignatova O.N., Kaganova I.I., Malyshev A.N., Podurets A.M., Rayevskii V.A., Skokov V.I., Tyupanova O.A. Mechanical properties of grade M1 copper before and after shock compression in a wide range of loading duration. Phys. Met. Metallogr., 2011, vol. 111, no. 2, pp. 197-206. DOI: 10.1134/S0031918X11010170.

9. Kolsky H. An investigation of the mechanical properties of material at very high rates of loading. In: Proc. Phys. Soc., London, 1949, vol. 62B, pp. 676-700.

10. Gallagher P.C.J. The influence of the alloying, temperature and related effects on the stacking fault energy. Met. Trans., 1970, vol. 1, no. 9, pp. 2429-2461.

11. Liu D., Miller A., Aust K.T. Annealing twin formation in a cast and annealed $\mathrm{Cu}-4$ at-percent Sn alloy. Canad. Met. Quart., 1984, vol. 23, no. 2, pp. 237-240.

12. Rodionova L.A., Popova E.N., Sudareva S.V., Vorob'eva A.E., Dergunova E.A., and Shikov A.K. Structure and mechanical properties of alloyed bronze matrix in Nb3Sn-based superconducting composites. Phys. Met. Metallogr., 1992, no. 73, pp. 71-77.

13. Popova E.N., Rodionova L.A., Sudareva S.V., Romanov E.P., Khlevova N.E., and Chukin A.M. Influence of different deformation techniques on the structure of bronze matrix in multifilamentary Nb3Sn composites. Phys. Met. Metallogr., 1993, no. 76, pp. 228-234.

14. Popov V.V., Stolbovsky A.V., Popova E.N., Pilyugin V.P. Structure and thermal stability of $\mathrm{Cu}$ after severe plastic deformation. Defect and Diffusion Forum, 2010, vols. 297-301, pp. 1312-1321. DOI: 10.4028/www.scientific.net/DDF.297-301.1312. 
Подана в журнал: 10.06 .2019

УДК 669.3'6:539.89:539.25

DOI: $10.17804 / 2410-9908.2019 .3 .041-051$

\title{
ЭВОЛЮЦИЯ СТРУКТУРЫ ОЛОВЯНИСТОЙ БРОНЗЫ И МЕДИ ПОСЛЕ ДИНАМИЧЕСКОГО СЖАТИЯ ПО МЕТОДУ КОЛЬСКОГО С ИСПОЛЬЗОВАНИЕМ РАЗРЕЗНОГО СТЕРЖНЯ ГОПКИНСОНА
}

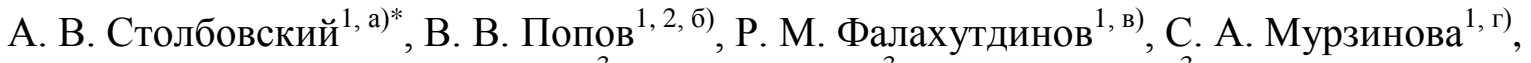 \\ Е. В. Шорохов ${ }^{3}$, 3. И. Завьялов ${ }^{3}$, А. А. Дегтярев ${ }^{3}$ \\ ${ }^{1}$ Институт физики металлов им. М.Н. Михеева УрО РАН, \\ ул. С. Ковалевской, 18, г. Екатеринбург, 620108, Российская Федерация \\ ${ }^{2}$ ФГБОУ ВО «Уральский государственный экономический университет», \\ 2. Екатеринбург, Российская Федерация \\ ${ }^{3}$ Российский Федеральный Ядерный Центр - ВНИИ технической физики, \\ 2. Снежинск, Российская Федерация \\ а) (ID \\ iD http://orcid.org/0000-0001-5228-7857 \\ iD http://orcid.org/0000-0002-9291-0102 \\ iD http://orcid.org/0000-0001-8505-9455 \\ iD http://orcid.org/0000-0001-5240-2966

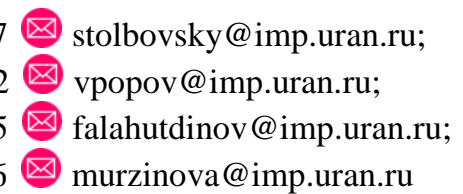 \\ *Ответственный автор. Электронная почта: stolbovsky@imp.uran.ru \\ Адрес для переписки: ул. С. Ковалевской, 18, Екатеринбург, 620108, Российская Федерация \\ Тел.: +7 (343) 378-35-74; факс: (343) 374-52-44
}

Исследовано влияние высокоскоростного динамического сжатия на структуру и механические свойства низколегированной оловянистой бронзы в различном исходном состоянии (крупнокристаллическое, с дополнительным отжигом, и литое, полученное посредством центробежного литья) и меди технической чистоты стандарта М1. Полученные результаты показывают, что динамическое сжатие образцов оловянистой бронзы в крупнокристаллическом исходном состоянии приводит, начиная со скорости деформирования $2,6 \cdot 10^{3} \cdot \mathrm{c}^{-1}$, к интенсивному развитию двойникования. В то же время при относительно небольших скоростях деформации динамическое сжатие образцов в исходном литом состоянии способствует повышению прочностных характеристик, а при дальнейшем повышении скорости деформации - к развитию релаксационных процессов. Однако дополнительный гомогенизирующий отжиг снижает вероятность протекания релаксационных процессов при нагружении, чем способствует получению максимально возможных механических характеристик. Испытания технически чистой меди M1 показали, что изменение скорости деформации значительно слабее влияет на свойства, чем в случае бронзы, и их значения ниже вследствие протекания релаксационных процессов как во время нагружения, так и после деформирования.

Ключевые слова: пластическая деформация, высокоскоростное деформирование, границы зерен, термическая стабильность, динамическое сжатие, метод Кольского, разрезной стержень Гопкинсона, оловянистая бронза, медь.

\section{1. Введение}

Эволюция структуры металлических материалов при пластическом деформировании различными методами является объектом многочисленных исследований. В настоящее время детально исследованы и качественно описаны процессы эволюции структуры низколегированных оловянистых бронз при пластической деформации статическими методами [1-2]. В последнее время появились исследования структуры металлов и сплавов, подвергнутых 
новому методу высокоскоростного деформирования - динамическому канально-угловому прессованию (ДКУП) [3-6]. Наряду с изучением возможности формирования нанокристаллической структуры методами интенсивной пластической деформации важной задачей при высокоскоростном деформировании является прогнозирование влияния высокоскоростных ударов, взрыва или других импульсных воздействий на структуру и свойства конструкционных материалов и изделий из них [7]. Несмотря на то, что изучение поведения материалов в условиях ударно-волнового нагружения является крайне важной научной и практической задачей, имеющиеся данные по этой проблеме немногочисленны, а полученные результаты неоднозначны [8].

В настоящей работе была поставлена задача расширить знания о влиянии высокоскоростного деформирования при воздействии импульсных нагрузок на низколегированную оловянистую бронзу и медь технической чистоты, чтобы оценить, насколько легирование меди $1 \%$ Sn влияет на свойства материала. Кроме того, была поставлена задача исследовать влияние исходного состояния низколегированной оловянистой бронзы (крупнокристаллическое, с дополнительным отжигом, и литое, полученное посредством центробежного литья) на последующее динамическое воздействие.

\section{2. Материал и методика исследования}

Для исследований использовалась оловянистая бронза, полученная методом сплавления меди технической чистоты стандарта M1 с присадкой олова в количестве 1 \% по массе, а также непосредственно медь технической чистоты, соответствующей требованиям стандарта M1. В табл. 1 представлен химический состав оловянистой бронзы.

Таблица 1 - Химический состав оловянистой бронзы $\mathrm{Cu}-1 \mathrm{Sn}$, вес. \%

\begin{tabular}{|c|c|c|c|c|c|c|}
\hline $\mathrm{Sn}$ & $\mathrm{Pb}$ & $\mathrm{Fe}$ & $\mathrm{Si}$ & $\mathrm{Ni}$ & $\mathrm{Zn}$ & $\mathrm{Bi}$ \\
\hline 1,05 & $\sim 0,01$ & $\sim 0,001$ & $<0,001$ & $\sim 0,001$ & $<0,001$ & $<0,001$ \\
\hline
\end{tabular}

Геометрические размеры образцов находились в пределах от 3 до 5 мм по высоте и от 6 до 10 мм в диаметре. Деформирование проводилось в условиях динамического сжатия по методу Кольского с использованием разрезного стержня Гопкинсона (РСГ) [9] при скоростях деформации $2-5 \cdot 10^{3} \mathrm{c}^{-1}$. Схема экспериментов по нагружению образцов приведена на рис. 1.

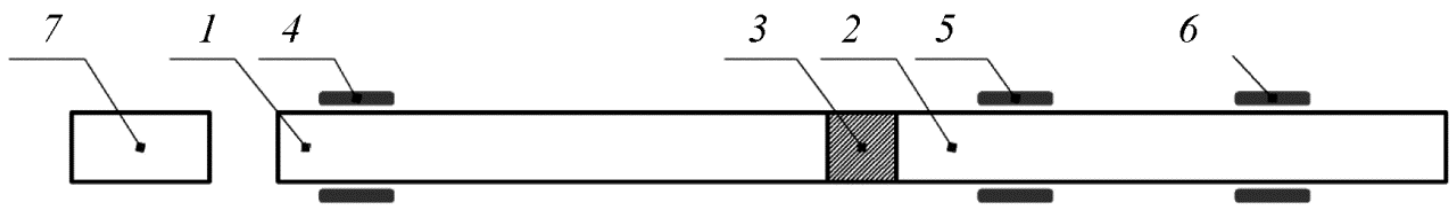

Рис. 1. Схема установки для динамических испытаний образцов:

1, 2 - нагружающий и опорный стержни соответственно; 3 - образец; 4, 5, 6 - тензодатчики; 7 - ударник

Импульсная нагрузка в нагружающем стержне создавалась ударником из высокопрочной стали, разгоняемым с помощью газовой пушки. В качестве нагружающего и опорного (мерных) стержней использовались стержни из высокопрочной стали. В опытах регистрировали импульсы деформации в нагружающем и опорном стержне с помощью тензорных датчиков.

В соответствии с теорией [9], при выводе соотношений для расчета напряжения $\sigma$ и деформации $\varepsilon$ образца предполагается, что поскольку он имеет малую длину, а время прохождения волны по длине образца существенно меньше длительности нагружающего им- 
пульса, то напряженно-деформированное состояние образца близко к однородному. Поэтому процесс упругопластического деформирования образца подобен квазистатическому, но протекающему с высокими скоростями деформации (до $\left.\sim 10^{4} \mathrm{c}^{-1}\right)$.

Параметрические зависимости $\sigma(t), \varepsilon(t)$ и $\dot{\varepsilon}(t)$ в образце определялись на основе экспериментальных записей упругой деформации в нагружающей $\varepsilon_{I}(t)$ и проходящей $\varepsilon_{T}(t)$ волнах напряжения (соответственно, в нагружающем и опорном стержнях) по следующим формулам [9]:

$$
\begin{aligned}
& \sigma(t)=\frac{E A}{A_{S}^{0}}\left[\varepsilon_{T}(t)\right] \\
& \varepsilon(t)=\frac{2 C}{L_{0}} \int_{0}^{t}\left[\varepsilon_{I}(t)-\varepsilon_{T}(t)\right] \cdot d t \\
& \dot{\varepsilon}(t)=\frac{2 C}{L_{0}}\left(\varepsilon_{I}(t)-\varepsilon_{T}(t)\right),
\end{aligned}
$$

где $A_{S}^{0}$ - исходная площадь поперечного сечения образца; $A$ - площадь поперечного сечения мерных стержней; $E$ - модуль упругости материала мерных стержней; $C$ - скорость продольных волн в стержнях; $L_{0}-$ первоначальная длина образца.

Bсе образцы были получены центробежным литьем и поделены на две группы: группа I - оловянистая бронза с крупнокристаллической структурой (размером кристаллитов около 1 мм), с дополнительным отжигом и без дополнительного отжига, группа II - крупнокристаллическая медь М1. Сводная таблица образцов, подвергнутых динамическим испытаниям

\begin{tabular}{|c|c|c|c|}
\hline № & Группа & $\begin{array}{l}\text { Материал } \\
\text { образца }\end{array}$ & $\begin{array}{c}\text { Наличие предварительного } \\
\text { отжига }\end{array}$ \\
\hline 1 & \multirow{3}{*}{ I } & \multirow{3}{*}{$\mathrm{Cu}-1 \% \mathrm{Sn}$} & Отсутствует \\
\hline 2 & & & $-\ll-$ \\
\hline 3 & & & Выполнен \\
\hline 4 & \multirow{3}{*}{ II } & \multirow{3}{*}{ M1 } & Отсутствует \\
\hline 5 & & & $-\ll-$ \\
\hline 6 & & & $-\ll-$ \\
\hline
\end{tabular}
по методу Кольского, представлена в табл. 2.

Таблица 2 - Сводная таблица образцов, подвергнутых испытаниям по методу Кольского

Образцы подвергали сжатию на установке РСГ при различных скоростях деформации, которые регулировали начальной скоростью полета ударника, что позволило получить условия деформирования в интервале скоростей деформации $(2 \div 5) \cdot 10^{3} \mathrm{c}^{-1}$.

Измерение микротвердости материала после динамического сжатия проводили на приставке к оптическому микроскопу Neophot-21 при нагрузке, эквивалентной 40 г, по методике, описанной в работе [1]. Микроструктуру изучали на этом же микроскопе.

\section{3. Результаты исследования и их обсуждение}

В первую группу вошли образцы оловянистой бронзы в крупнокристаллическом со-

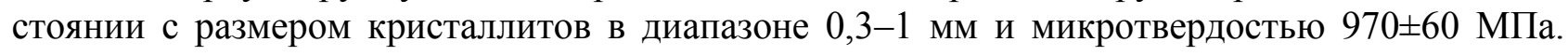


На образце 3 был дополнительно проведен гомогенизирующий отжиг для определения его влияния на результат последующего импульсного нагружения. Этот отжиг не произвел существенного влияния на исходные параметры микроструктуры и микротвердость, значение которой в образце 3 составляет $960 \pm 40$ МПа. Можно отметить, что в отожженном образце зеренная структура несколько более равномерная по размерам, а кроме того, появляются редкие двойники отжига - тонкие, прямые и одиночные.

Необходимо отметить, что во всех образцах состава $\mathrm{Cu}-1$ \% $\mathrm{Sn}$ наблюдалась газовая пористость, связанная, по-видимому, с технологией получения образцов методом сплавления меди технической чистоты стандарта M1 с присадкой олова. Средний размер газовых пор составлял $9 \pm 5$ мкм.

Степень и скорость деформации, динамический условный предел текучести, временное сопротивление разрушению и микротвердость образцов группы I приведены в табл. 3. Условный предел текучести и временное сопротивление разрушению были определены на основании диаграмм напряжение-деформация, полученных на установке РСГ.

Таблица 3 - Механические свойства образцов сплавов $\mathrm{Cu}-1$ \% Sn (группа I)

\begin{tabular}{|c|c|c|c|c|c|c|}
\hline \multirow{2}{*}{ № } & \multirow{2}{*}{$\begin{array}{c}\text { Материал } \\
\text { образца }\end{array}$} & $\varepsilon, \%$ & $\dot{\varepsilon}, 10^{3} \cdot \mathrm{c}^{-1}$ & $\sigma_{0,2}, \mathrm{M \Pi а}$ & $\sigma_{\text {в }}$, МПа & $H$, МПа \\
\cline { 1 - 5 } 1 & \multirow{3}{*}{$\mathrm{Cu}-1 \%$ Sn } & 19,79 & 2,0 & - & 330 & $1460 \pm 90$ \\
\cline { 1 - 5 } & & 22,06 & 2,6 & - & 340 & $1340 \pm 60$ \\
\cline { 1 - 5 } & & 30,72 & 3,3 & 91 & 380 & $1600 \pm 130$ \\
\hline
\end{tabular}

По сравнению с исходным состоянием микротвердость в образцах 1 и 2 после деформации увеличилась на 40-50 \%. С увеличением степени и скорости деформации можно было ожидать существенного повышения прочностных характеристик и твердости образцов. Однако, исходя из данных, приведенных в табл. 3, это не совсем так. В образцах 1 и 2, отличающихся небольшим увеличением степени деформации (на 10 \%) и существенным увеличением скорости деформирования (в 1,3 раза), временное сопротивление разрушению увеличилось незначительно (на 3 \%), а микротвердость не только не возросла, а даже уменьшилась. При более существенном увеличении степени деформации (еще на 40 \%) и ее скорости еще в 1,3 раза (в образце 3 по сравнению с образцом 2), временное сопротивление разрушению увеличилось на $12 \%$, а микротвердость - на $19 \%$.

При этом следует напомнить, что образец 3 перед деформацией подвергался отжигу, который, как отмечено выше, не оказал заметного влияния на микроструктуру и привел к незначительному понижению микротвердости.

Чтобы объяснить полученные результаты, были проведены структурные исследования. Микроструктура образцов серии І представлена на рис. 2. Во всех образцах сохранились поры, которые наблюдались и в исходном состоянии. Структура всех образцов крупнозернистая, с размерами зерен от 300 мкм до 1 мм, т. е. при высокоскоростной деформации этим методом не произошло существенного измельчения структуры. 


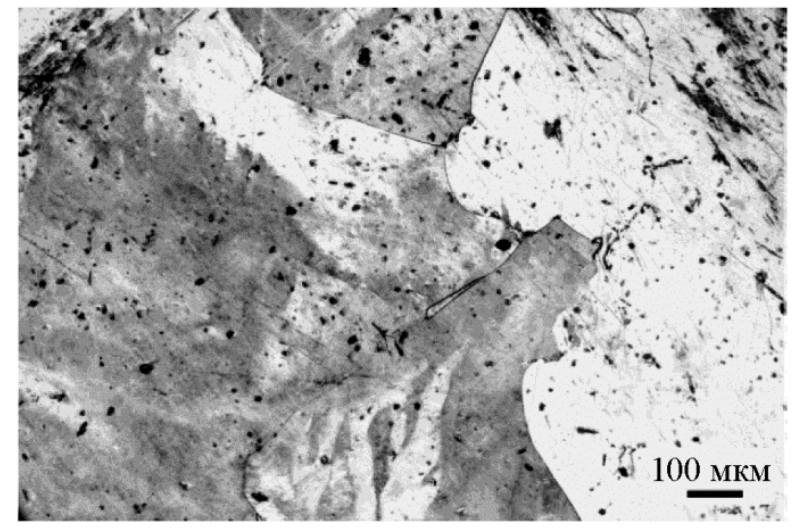

$a$

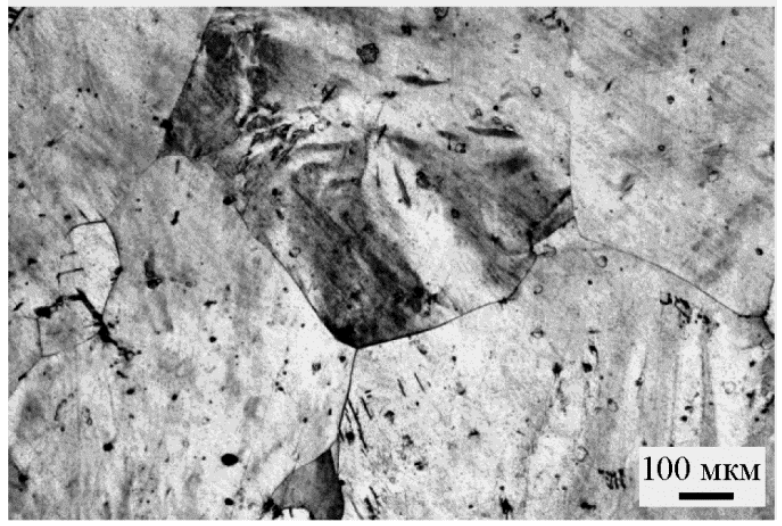

$\sigma$

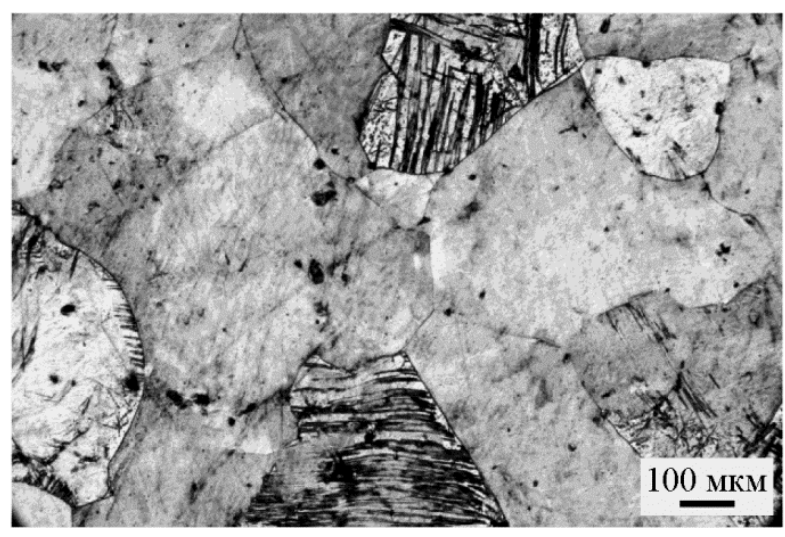

B

Рис. 2. Микрофотографии структуры образцов группы I (Cu-1\% $\mathrm{Sn})$ : $a$ - образец $1 ; \sigma$ - образец $2 ; в$ - образец 3

Как видно из рис. 2 , в образце 1 , деформированном со скоростью $2,0 \cdot 10^{3} \mathrm{c}^{-1}$, в структуре отсутствуют двойники. Границы зерен изогнутые и не очень четкие, разброс зерен по размерам такой же большой, как в исходном состоянии, поры сохранились по всему образцу. В некоторых зернах видны следы дендритов, унаследованные от исходного литого состояния. С увеличением скорости деформации до $2,6 \cdot 10^{3} \mathrm{c}^{-1}$ (образец 2) границы зерен становятся более четкими, разброс зерен по размерам уменьшается. В некоторых зернах появляются тонкие прямые двойники, но общее их количество невелико, поэтому можно заключить, что основной механизм деформации в обоих случаях дислокационный.

При дальнейшем повышении скорости деформирования и степени деформации (образец 3) границы зерен становятся более прямыми и четкими, происходит небольшое измельчение зерен, структура в целом более равномерная. Основное же отличие этого образца в том, что в нем интенсивно развивается двойникование и в структуре наблюдается большое количество двойников, причем по нескольким системам двойникования. Хотя образец 3 отличается от образцов 1 и 2 не только более высокой степенью и скоростью деформации, но и тем, что он подвергался предварительному отжигу, количество двойников отжига в нем в исходном состоянии было настолько незначительным, что двойникование является результатом не отжига, а достаточно интенсивной деформации.

Склонность оловянистых бронз к двойникованию при деформации и отжиге обусловлена тем, что олово понижает энергию дефекта упаковки (ЭДУ) медных сплавов [10-11]. В меди и ее сплавах плоскостями двойникования являются плотноупакованные плоскости типа $\{111\}$ си, и в определенных условиях возможно двойникование сразу по нескольким си- 
стемам, особенно при легировании оловом даже в небольшом количестве. Ранее было обнаружено, что в стесненных условиях, например, при волочении композитной проволоки $\mathrm{Cu}-\mathrm{Sn} / \mathrm{Nb}$, появление многочисленных двойников в бронзе обусловлено изменением преимущественного механизма ее деформации со скольжения дислокаций на двойникование $[12,13]$. Интенсивное двойникование развивается в бронзах и при динамическом канальноугловом прессовании [6]. При динамическом сжатии по методу Кольского скорость и степень деформации существенно меньше, чем при ДКУП, но при достаточно высокой степени и скорости деформации этим методом двойникование тоже интенсивно развивается.

Сравнение механических свойств образцов серии I показывает, что все измеренные характеристики образца 3 существенно выше, чем образцов 1 и 2, а микротвердость достигает значения $1600 \pm 130$ МПа. Такое значительное увеличение микротвердости при увеличении скорости деформации динамическим сжатием, скорее всего, связано с увеличением дисперсности структуры и плотности дефектов кристаллического строения. В то же время дополнительный гомогенизирующий отжиг снизил вероятность протекания при нагружении релаксационных процессов, чем способствовал получению максимально возможных механических характеристик. Тем не менее, они значительно ниже, чем после ДКУП (1820-1960 МПа после 1 прохода) [6].

Чтобы оценить, насколько легирование меди оловом в количестве 1 \% влияет на свойства и их эволюцию при деформации, были проведены испытания чистой меди М1 этим же методом. Механические характеристики, определенные из диаграмм напряжение-деформация, и микротвердость образцов группы II приведены в табл. 4.

Таблица 4 - Механические свойства образцов меди М1 (группа II)

\begin{tabular}{|c|c|c|c|c|c|c|}
\hline № & $\begin{array}{c}\text { Материал } \\
\text { образца }\end{array}$ & $\varepsilon, \%$ & $\dot{\varepsilon}, 10^{3} \cdot \mathrm{c}^{-1}$ & $\sigma_{0,2}, \mathrm{M \Pi а}$ & $\sigma_{\text {в }}$, МПа & Н, МПа \\
\hline 4 & \multirow{3}{*}{$\mathrm{Cu}$ M1 } & 28,51 & 2,1 & 46 & 240 & $1290 \pm 70$ \\
\hline 5 & & 37,02 & 2,8 & 55 & 270 & $1180 \pm 60$ \\
\hline 6 & & 32,19 & 3,3 & 90 & 290 & $1220 \pm 20$ \\
\hline
\end{tabular}

Как и можно было ожидать, при всех исследованных скоростях и степенях деформации механические свойства бронзы (временное сопротивление разрушению, микротвердость) выше, чем у технически чистой меди. При этом поведение при деформации меди и бронзы отличается. По сравнению с исходным состоянием, в бронзе максимальный прирост микротвердости (на 50 \%) наблюдался при самой высокой степени и скорости деформации. В меди микротвердость увеличилась на 36 \% при минимальной скорости и степени динамического сжатия (образец 4), но ее прирост уменьшился до 24 \% при максимальной степени деформации (образец 5) и до 28 \% - при самой высокой скорости (образец 6). Такое поведение меди свидетельствует о преобладании в ней релаксационных процессов при изучаемом способе деформации.

Сравним влияние скорости деформации на механические характеристики меди M1 (группа II) и бронзы (группа I). В обоих случаях с увеличением скорости происходит увеличение динамического предела прочности, но в меди эта зависимость становится слабее с увеличением скорости, в то время как в бронзе она, наоборот, усиливается. Вначале в меди происходит более быстрое повышение $\sigma_{\text {в }}$ (на 12,5\% при увеличении скорости в 1,3 раза по сравнению с 3 \% у бронзы), а затем оно замедляется и составляет 7 \% (по сравнению с $12 \%$ у бронзы), т. е. в меди достигается насыщение.

Микротвердость меди с увеличением степени и скорости деформации меняется не систематически, и изменения ее не превышают погрешности измерений, что указывает на протекание релаксационных процессов в процессе и после нагружения. Такое поведение 
меди при интенсивной пластической деформации весьма ожидаемо и наблюдалось ранее при кручении под высоким давлением [13].

Металлографические снимки структуры образцов 4 и 6 представлены на рис. 3. При изменении скорости деформации зеренная структура изменилась незначительно. Несмотря на то, что полученные значения микротвердости выше, чем у исходного состояния до нагружения $(950 \pm 60 \mathrm{MПа)}$, существенных изменений в размерах кристаллитов не обнаружено. Единственное различие в образцах группы II после нагружения состоит в том, что при минимальной скорости деформации $\left(2,1 \cdot 10^{3} \mathrm{c}^{-1}\right)$ границы зерен преимущественно прямые, тогда как при максимальной скорости $\left(3,3 \cdot 10^{3} \mathrm{c}^{-1}\right)$ видны искривленные границы зерен, характерные для деформированного состояния. По-видимому, границы зерен в меди более подвижны, чем в бронзе.

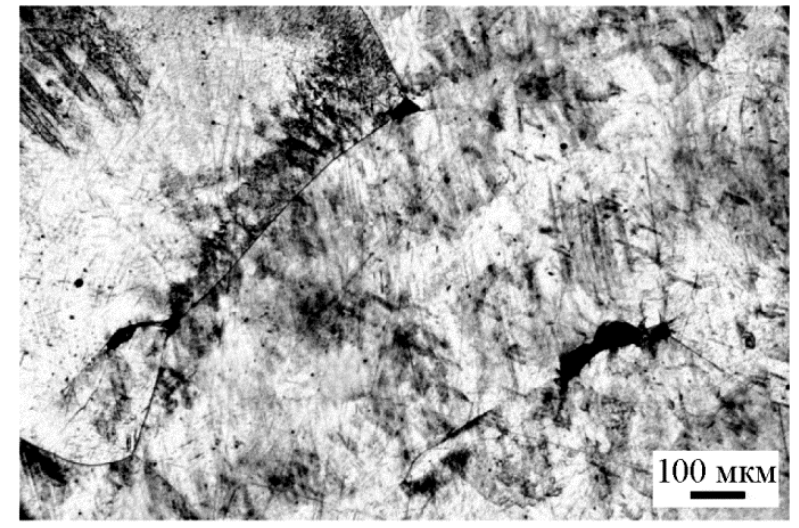

$a$

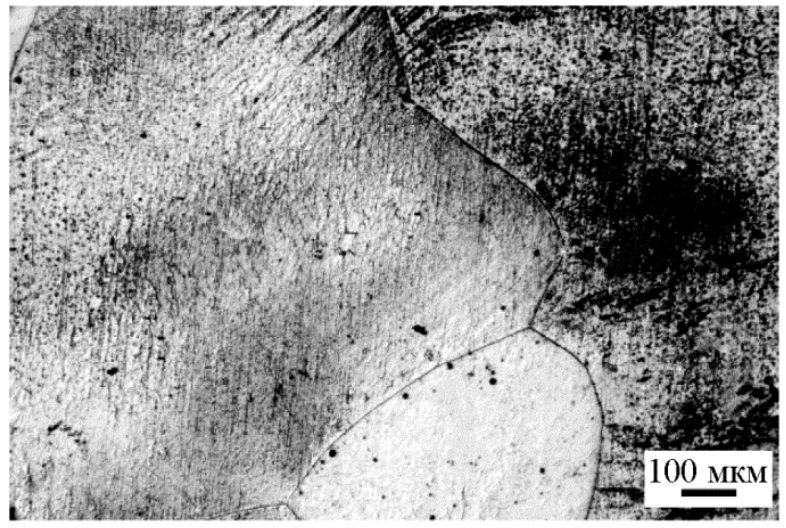

$\sigma$

Рис. 3. Микрофотографии структуры образцов группы II (Cu): $a$ - образец $4 ; \sigma$ - образец 6

На рис. 4 представлены зависимости микротвердости от скорости деформации образцов меди и бронзы. Видно, что эти зависимости не являются монотонными. Для бронзы образец с предварительным отжигом вынесен отдельной точкой. Возможно, немонотонное изменение микротвердости с увеличением скорости связано с нагревом материала при деформации. Это вполне вероятно, поскольку известно, что при ударном сжатии происходит разогрев материала, вследствие которого возможно протекание релаксационных процессов.

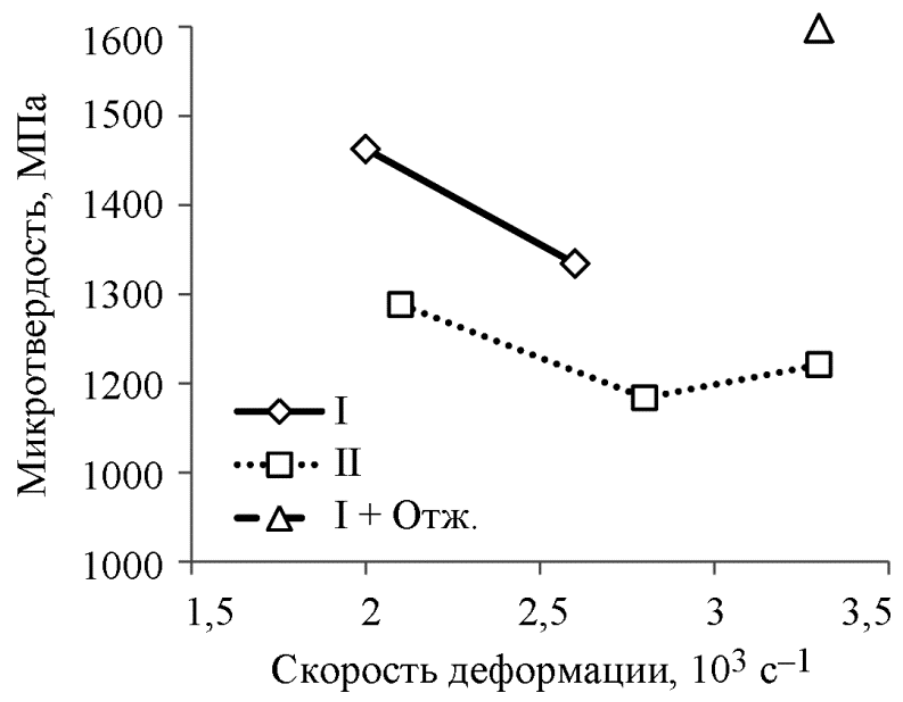

Рис. 4. Зависимость микротвердости от скорости деформации образцов 
При этом следует отметить немонотонное изменение и других механических характеристик (табл. 3 и 4), что также указывает на протекание релаксационных процессов именно во время нагружения. Эти процессы более выражены в меди, чем в бронзе, и препятствуют измельчению структуры при деформации.

Полученные результаты показывают, что динамическое сжатие образцов меди и бронзы в исходном литом состоянии способствует повышению прочностных характеристик, причем в бронзе это повышение нарастает с увеличением скорости, а в меди уменьшается из-за развития релаксационных процессов. В бронзе с увеличением степени и скорости деформации включается механизм двойникования, хотя оно выражено слабее, чем при динамическом канально-угловом прессовании.

\section{4. Заключение}

Исследовано влияние высокоскоростного сжатия на структуру и механические свойства низколегированной оловянистой бронзы и меди технической чистоты стандарта М1.

Обнаружено, что в исходно крупнозернистой оловянистой бронзе с увеличением скорости деформации происходит изменение механизма деформации с дислокационного на двойниковый. Двойникование интенсивно развивается, начиная со скорости деформирования 2,6 $10^{3} \cdot \mathrm{c}^{-1}$. Самые высокие значения предела прочности (380 МПа) и микротвердости (1600 МПа) достигнуты при максимальной исследованной скорости нагружения $\left(3,3 \cdot 10^{3} \cdot \mathrm{c}^{-1}\right)$. По сравнению с исходным состоянием прирост микротвердости составляет $30 \%$, что обусловлено именно двойникованием, поскольку заметного измельчения зеренной структуры не обнаружено.

В меди М1, в отличие от бронзы, влияние скорости деформации на прочностные свойства ослабляется с ростом этого параметра, что объясняется протеканием релаксационных процессов при динамическом сжатии и после его окончания. Это подтверждается результатами структурных исследований, которые показали, что при изменении скорости деформации зеренная структура меди изменяется незначительно.

Таким образом, на структуру и свойства меди и бронзы при динамическом сжатии по методу Кольского влияют два конкурирующих фактора - деформационное упрочнение и релаксационные процессы. В меди преобладают процессы релаксации, которые ослабляют влияние высокоскоростной деформации на структуру и механические характеристики. В бронзе с ростом скорости деформирования включается механизм двойникования, релаксационные процессы подавляются и достигается более высокое упрочнение.

\section{Благодарность}

Работа выполнена в рамках государственного задания МИНОБРНАУКИ России (тема «Функиия», номер госрегистрачии № АAАA-A19-119012990095-0) при поддержке программы фундаментальных исследований УрО РАН (проект № 18-10-2-37).

\section{Литература}

1. Stolbovsky A. V., Popov V. V., Popova E. N. Structure and Thermal Stability of Tin Bronze Nanostructured by High Pressure Torsion // Diagnostics, Resource and Mechanics of materials and structures. - 2015. - Iss. 5. - P. 118-132. - DOI: 10.17804/2410-9908.2015.5.118-132. URL: http://dream-journal.org/issues/2015-5/2015-5_52.html (accessed: 30.10.2017).

2. Effect of Severe Plastic Deformation by High-Pressure Torsion on the Structure and Properties of Copper and Tin Bronze / A. V. Stolbovsky, V. V. Popov, E. N. Popova, R. M. Falahutdinov // Diagnostics, Resource and Mechanics of materials and structures. - 2017. - Iss. 5. - P. 13-22. - 
DOI: 10.17804/2410-9908.2017.5.013-022. - URL: http://dream-journal.org/issues/2017-5/20175_144.html (accessed: 09.07.2018).

3. Structure Formation in Copper during Dynamic Channel_Angular Pressing / I. V. Khomskaya, V. I. Zel'dovich, E. V. Shorokhov, N. Yu. Frolova, I. N. Zhgilev, A. E. Kheifets // Phys. Met. Metallogr. - 2008. - Vol. 105, no. 6. - P. 586-593. - DOI: 10.1134/S0031918X08060094.

4. High Speed Deformation of Metallic Materials Using Channel Angular Pressing for Producing an Ultrafine Grained Structure / E. V. Shorokhov, I. N. Zhgilev, I. V. Khomskaya, I. G. Brodova, V. I. Gurov, N. P. Oglezneva, I. G. Shirinkina, A. E. Kheifets, V. V. Astaf'ev // Russ. Metall. (Metally). - 2010. - No. 4. - P. 323-327. - DOI: 10.1134/S0036029510040142.

5. Zel'dovich, D. V. Gunderov, N. Yu. Frolova, A. A. Evolution of the Structure upon Heating of Submicrocrystalline and Nanocrystalline Copper Produced by High Rate Deformation / I. V. Khomskaya, V. I. Zel'dovich, A. E. Kheifets, N. Yu. Frolova, V. P. Dyakina, V. A. Kazantsev // Phys. Met. Metallogr. - 2011. - Vol. 111, no. 4. - P. 367-374. - DOI: 10.1134/S0031918X11030070.

6. Особенности деформационного поведения бронз при динамическом канальноугловом прессовании / А. В. Столбовский, В. В. Попов, Е. Н. Попова, Р. М. Фалахутдинов, Е. В. Шорохов // Международный журнал прикладных и фундаментальных исследований. 2016. - № 11. - C. 858-863.

7. Petrova A. N., Brodova I. G., Razorenov S. V. Strength properties and structure of a submicrocrystalline $\mathrm{Al}-\mathrm{Mg}-\mathrm{Mn}$ alloy under shock compression // Phys. Met. Metallogr. 2017. - Vol. 118, no. 6. - P. 601-607. - DOI: https://doi.org/10.1134/S0031918X17060072.

8. Mechanical properties of grade M1 copper before and after shock compression in a wide range of loading duration / G. V. Garkushin, G. E. Ivanchikhina, S. V. Razorenov, O. N. Ignatova, I. I. Kaganova, A. N. Malyshev, A. M. Podurets, V. A. Rayevskii, V. I. Skokov, O. A. Tyupanova // Phys. Met. Metallogr. - 2011. - Vol. 111, no. 2. - P. 197-206. - DOI: 10.1134/S0031918X11010170.

9. Kolsky H. An investigation of the mechanical properties of material at very high rates of loading // Proc. Phys. Soc., London. - 1949. - Vol. 62B. - P. 676-700.

10. Gallagher P. C. J. The influence of the alloying, temperature and related effects on the stacking fault energy // Met. Trans. - 1970. - Vol. 1, no. 9. - P. 2429-2461.

11. Liu D., Miller A., Aust K. T. Annealing twin formation in a cast and annealed $\mathrm{Cu}-4$ at-percent Sn alloy // Canad. Met. Quart. - 1984. - Vol. 23, no. 2. - P. 237-240.

12. Structure and mechanical properties of alloyed bronze matrix in Nb3Sn-based superconducting composites / L. A. Rodionova, E. N. Popova, S. V. Sudareva, A. E. Vorob'eva, E. A. Dergunova, and A. K. Shikov // Phys. Met. Metallogr. - 1992. - No. 73. -P. 71-77.

13. Influence of different deformation techniques on the structure of bronze matrix in multifilamentary Nb3Sn composites / E. N. Popova, L. A. Rodionova, S. V. Sudareva, E. P. Romanov, N. E. Khlevova, and A. M. Chukin // Phys. Met. Metallogr. - 1993. - No. 76. - P. 228-234.

14. Structure and thermal stability of $\mathrm{Cu}$ after severe plastic deformation / V. V. Popov, A. V. Stolbovsky, E. N. Popova, V. P. Pilyugin // Defect and Diffusion Forum. - 2010. - Vol. 297-301. P. 1312-1321. - DOI: 10.4028/www.scientific.net/DDF.297-301.1312. 